\title{
EXCITATION CROSS SECTION FOR SOME OF THE DOUBLY IONIZED STATES OF ARGON, NEON AND KRYPTON BY FAST ELECTRONS
}

S.H. Koozekanani

\section{The Ohio State University \\ ElectroScience Laboratory \\ (formerly Antenna Laborotory) \\ Department of Electrical Engineering \\ Columbus, Ohio $\mathbf{4 3 2 1 2}$}

REPOR T 1093-36

8 January 1968

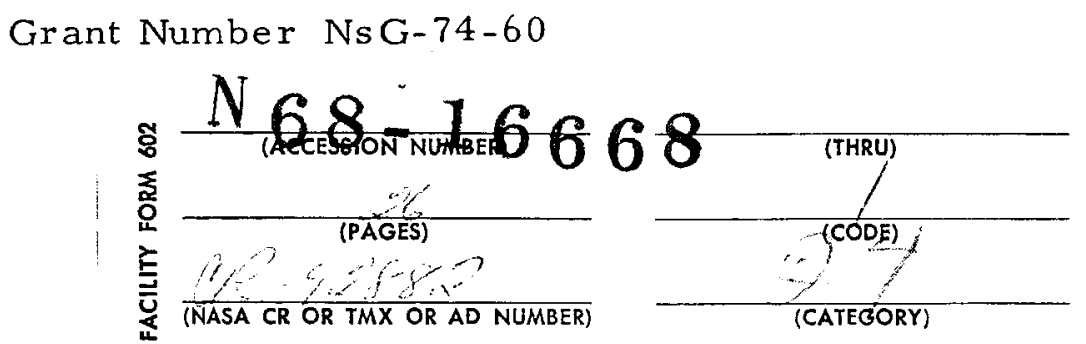

National Aeronautics and Space Administration

Office of Grants and Research Contracts

Washington, D.C. 20546 


\section{REPORT}

by

The Ohio State University ElectroScience Laboratory

(Formerly Antenna Laboratory)

Columbus, Ohio 43212

Sponsor

Grant Number

Investigation of

Subject of Report

Submitted by
National Aeronautics and Space Administration Office of Grants and Research Contracts Washington, D.C. 20546

Ns G-74-60

Receiver Techniques and Detectors for Use at Millimeter and Submillimeter Wave Lengths

Excitation Cross Section for Some of the Doubly Ionized States of Argon, Neon and Krypton by Fast Electrons

S.H. Koozekanani

ElectroScience Laboratory

Department of Electrical Engineering

Date

8 January 1968 


\section{ABSTRACT}

Excitation cross section for the doubly ionized states of argon, neon and krypton has been found for the process where a fast moving free electron impinges upon a neutral atom causing the neutral atom to go from $\ell^{N}$ to ( $\left.\ell^{N-3}-n \ell^{\prime \prime}\right)$ in a very short timie compared to the relaxation time of the ion. 
I. INTRODUCTION 1

II. CALCULATION 2

Case One

Case Two

111. CONCLUSIONS 12

$\begin{array}{ll}\text { REFERENCES } & 13\end{array}$

ACKNOW LEDGEMENT 14 


\section{EXCITATION CROSS SECTION FOR SOME OF THE DOUBLY IONIZED STATES OF ARGON, NEON AND KRYPTON BY FAST ELECTRONS}

\section{INTRODUCTION}

It has been shown $n^{1,2,3}$ that the method of sudden perturbation can account for the excitation of some of the excited states of argon II in the pulsed argon laser. This method of calculation yields correct results only when the Hamiltonian changes from an initial state to a final state in a very short time compared to the relaxation time of the system. 5 In this method, the eigenfunctions of the unperturbed Hamiltonian are expanded in terms of the eigenfunctions of the perturbed system. In the problem that we are considering, we assume that a neutral atom with an $\ell N$ outer shell configuration loses $m(m=2)$ of its $\ell N$ electrons, in a very short time compared to the relaxation time of the atom, after making a collision with a fast impinging electron. Before collision, the eigenfunction of the system consists of the product of a free electron wave function with that of the product of a free electron wave function with that of the bound atomic electrons. After collision, the new eigenfunctions are the product of the wave function of $(m+l)$ free electron, with those of $\ell^{\mathrm{N}-\mathrm{m}}$ bound atomic electrons. Two cases are considered. For the first case, it is assumed that the atom makes a collision with a fast-moving electron, loses two of its $\mathrm{N}$ outer shell electrons, and is simultaneously excited, i.e., $\ell N \rightarrow\left(\ell_{n} N-3-n \ell\right)$. There the atom is doubly ionized and excited at the same time. For the second case, it is assumed that two collisions are involved, i.e., the atom makes a collision and goes to an excited single ionized configuration $\left(\ell_{1 n} N-2_{-n} \ell^{\prime}\right)$; then, though another collision, it may lose its excited $n \ell^{\prime}$ electron or one of the $\ell_{n} N-2$ core electrons. This process is important when $\left(\ell_{1 n} N-2-n \ell^{\prime}\right)$ is a metzstable state or a state with relatively long life time. The states for which excitation cross sections will be found are $\left|\ell^{3}, \phi_{2}, \mathrm{n} \ell \mathrm{s}^{\prime \prime} ;{ }^{3} \mathrm{P}_{\mathrm{J}}>,\right| \ell^{3}, \phi_{2}, \mathrm{n} \ell \mathrm{s}^{\prime \prime}$, ${ }^{1} \mathrm{D}_{2}>$ and $\mid \ell^{3}, \phi_{2}, \mathrm{n} \ell \mathrm{S} ;{ }^{1} \mathrm{~S}_{\mathrm{O}}>$ with $\phi_{2}={ }^{4} \mathrm{~S},{ }^{2} \mathrm{D}$ or ${ }^{2} \mathrm{P}$.

To obtain the probability of production of any one of the new states, we have to expend the unperturbed Hamiltonian eigenfunctions in terms of the complete set of eigenfunctions of the new Hamiltonian. The overlap integrals in these calculations involve those between electronic bound states before and after collision and between bound and resulting free electronic states. The absolute-square values of the former set of integrals are proportional to the excitation cross section, whereas the corresponding values for the latter set are proportional to the ionization cross section. Since experimental double ionization cross sections for 
most of the atoms are known, the excitation cross section for case one can be calculated exactly.

For example, for the $3 \mathrm{p}^{6}$ outershell configuration of neutral argon to go to the $3 \mathrm{p}^{3}-4 \mathrm{p}$ state after collision with a $100 \mathrm{ev}$ electron, we find a cross section of $8.9 \times 10^{-19} \mathrm{~cm}^{2}$. In what follows we shall attempt to find expressions for the cross sections of the various excited states. These states are represented by their angular momentum quantum numbers, i.e., states of the form $\mid \ell N-3 \phi_{2}, n \ell$ "s; $\phi_{1}>$. For noble gas atoms where $N=6, \ell=\ell{ }^{\prime \prime}=1$ we have $\phi_{2} \equiv 4 S,{ }^{2} \mathrm{P}$ or ${ }^{2} \mathrm{D}$ and $\phi_{1}$ can be ${ }^{1} S_{1},{ }^{3} P_{0,1,2}$ or ${ }^{1} D_{2}$. We shall calculate cross sections for all the 12 possible combinations of $\phi_{2}$ and $\phi_{1}$. Figure 1 .

II. CALCULATION

\section{Case One}

We now assume that the atom makes a collision with an electron and loses two of its electrons, simultaneously. In order to know the possible excited states, we have to expand the unperturbed eigenfunctions $1 \ell N_{\phi_{0}} ; F_{1 i}>$ in terms of the eigenfunctions of the perturbed Hamiltonian. This eigenfunction consists of the product of $\left|\ell^{N_{\phi_{O}}}\right\rangle$ bound wave function and the $F_{1 i}$ free electron wave function. We have, after expansion

$$
\left|\ell N_{\phi_{0}} ; F_{1 i}\right\rangle=\sum_{n, f} a_{n f}\left|\ell \frac{N-3}{n} \phi_{2} ; n \ell \ell^{\prime \prime}, \phi_{1}^{\prime}: F_{1 f}, F_{2 f}, F_{3 f}\right\rangle
$$

where $\mid \ell \frac{N}{N-3} \phi_{2} ; n \ell^{\prime \prime} s, \phi_{1}{ }^{\prime}>$ is an excited doubly ionized state of the atom, $n l^{\prime \prime}$ is the excited electron, and $\ell_{n}$ are the core electrons. The subscript $n$ indicates that the radial wave function of $\ell_{n}^{N-3}$ core electrons changes as the principal quantum number $n$ of the excited electron assumes different values. In Eq. (1a), s stands for the spin 1,2 of the electron, and $\phi$ with or without super-or subscript stands for the corresponding angular wave functions. For example, in $\ell N-3 \phi_{2}, \phi_{2}$ replaces $L_{2}, S_{2}, M_{L_{2}}$ and $N_{S_{2}}$, where $L_{2}$ is the total orbital angular momentum, $S_{2}$ is the total spin-angular momentum, and $\mathrm{ML}_{2}$ and $\mathrm{NS}_{2}$ are the projections of $L$ and $S$ on the magnetic $z$-axis. $F_{1 f}, F_{2 f}$ and $F_{3} f$ represent the wave functions for the three free electrons after the impact. The summation in Eq. (la) is over all the possible final states, F. For Eq. (1) we have 
(lb)

$$
\sum_{f} a_{n f}=\sum_{f}<\ell{ }_{n}^{N-3} \phi_{2}, n \ell^{\prime \prime} s, \phi_{1}^{\prime}: F_{1 f}, F_{2 f}, F_{3 f} \mid \ell N_{\phi_{0}}: F_{1 \mathfrak{f}}>.
$$

To calculate these inetrix elements, we first expand the bound atomic wave function $\mid \ell N_{\phi_{O}}>$ in terms of its parents and grandparents and then decouple two electrons, in other words we have

$$
\begin{gathered}
\left|\ell^{N_{\phi_{0}}}\right\rangle=\sum_{\phi, \phi_{1}}\left(\ell N-1_{\phi}\left\{\mid \ell^{N_{\phi}}\right)\left(\ell \ell^{N-2} \phi_{1}\left\{\mid \ell^{N-1} \phi\right)\right.\right. \\
\mid \ell^{N-2} \phi ; \ell s ; \ell s, \phi_{0}>
\end{gathered}
$$

where two electrons have been separated from the core. The eigenfunctions in Eq. (2a) can be decoupled to give us,

$$
\begin{aligned}
& \left.\ell_{\ell} \mathrm{N}_{\phi_{\mathrm{O}}}\right\rangle=\sum \\
& (-)^{L+L_{1}-2 \ell+M_{L_{O}}+M_{L}+S+S_{1}-2 S+N S_{O}+N S} \\
& \phi, \phi_{1}, M_{L}, M_{\ell}, M_{L_{1}} \text {, } \\
& \mathrm{N}_{S}, \mathrm{~N}_{\mathrm{S}}, \mathrm{N}_{\mathrm{S}_{1}}, \mathrm{Ml}_{2}, \mathrm{~N}_{\mathrm{S}_{2}} \\
& \left\{\left[L_{O}\right][L]\left[S_{O}\right][S]\right\}^{\frac{1}{2}}\left(\ell ^ { N - 1 } \phi \{ | \ell _ { O _ { O } } ) \left(\ell N-2_{\phi_{1}}\left\{\mid \ell^{N-1} \phi\right)\right.\right. \\
& \left(\begin{array}{ccc}
L & \ell & L_{O} \\
M_{L} & M_{\ell_{1}} & M_{L_{O}}
\end{array}\right)\left(\begin{array}{ccc}
L_{1} & \ell & L \\
M_{L_{1}} & M_{\ell_{2}} & M_{L}
\end{array}\right)\left(\begin{array}{ccc}
S & s & S_{O} \\
N_{S} & N_{S_{1}} & N_{S_{O}}
\end{array}\right)\left(\begin{array}{ccc}
S_{1} & s & S \\
N_{S_{1}} & N_{S_{2}} & N_{S}
\end{array}\right) \\
& \left.\left|\ell^{\mathrm{N}-2_{\phi_{1}}}\right\rangle\left|\ell_{S}, \mathrm{M}_{\ell_{1}} \mathrm{~N}_{\mathrm{S}_{1}}>\right| \ell_{\mathrm{S}}, \mathrm{M}_{\ell_{2}} \mathrm{~N}_{\mathrm{S}_{2}}\right\rangle
\end{aligned}
$$

where the symbol $[x]=2 x+1$, $\phi_{n}$ stands for $L_{n}, S_{n}, M_{L_{n}}$ and $N_{S_{n}}$. Replacing Eq. (2a) back in Eq. (1) we obtain 
(2c)

$$
\begin{aligned}
& \sum_{\mathrm{f}} a_{n f}=\sum_{\phi, \phi_{1}}\left(\ell N-2_{\phi_{1}}\left\{\mid \ell N-l_{\phi}\right)\left(\ell N-1+\left\{\mid \ell N_{\phi_{0}}\right)\right.\right. \\
& <\ell \stackrel{\mathrm{N}-3}{\mathrm{\phi}} \phi_{2}, \mathrm{nl} \ell^{\prime \prime} \mathrm{s}, \phi_{1}{ }^{\prime} \mid \ell^{\mathrm{N}-2_{\phi_{1}}}> \\
& \times \int(-1)^{L+L_{1}-2 l+M_{L}}+M_{L}+S+S_{1}-2 s+N_{S_{O}}+N_{S} \\
& \mathrm{M}_{L_{1}}, \mathrm{M}_{\ell_{1}}, \mathrm{M}_{L_{1}}, \mathrm{M}_{\ell_{2}} \\
& \mathrm{~N}_{S}, \mathrm{~N}_{\mathrm{S}}, \mathrm{N}_{\mathrm{S}_{1}}, \mathrm{NS}_{\mathrm{S}}, \mathrm{f} \\
& \left\{\left[\mathrm{L}_{\mathrm{O}}\right][\mathrm{L}]\left[\mathrm{S}_{\mathrm{O}}\right][\mathrm{S}]\right\}^{\frac{1}{2}}
\end{aligned}
$$

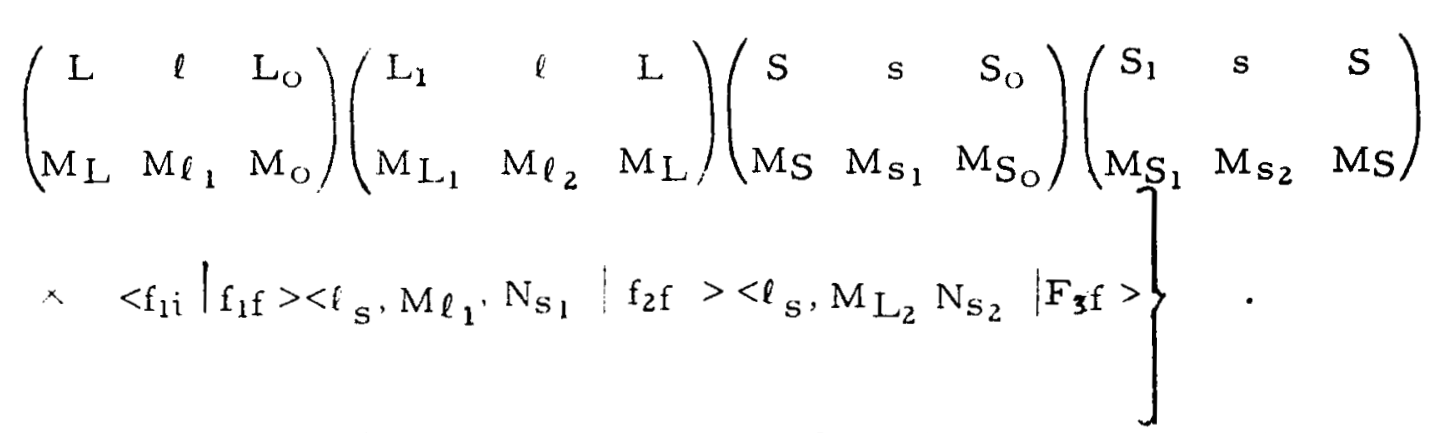

In the above equation the absolute square value of the quantity in braces $(\{\})$ is proportional to the durble ionization probability of the atom. It consists of the product of an initially free electron and two bound electrons with the wave functions of three free electrons. In Eq. (2c), one more matrix element remains to be calculated, and that is the quantity

$$
\begin{aligned}
& \left\langle\ell n^{N-3} \phi_{2} ; n \ell^{\prime \prime}, o_{1}^{\prime}\right| \ell^{N-2_{\phi_{1}}}> \\
& =\sum(-1)^{L_{2}+L_{2}{ }^{\prime}-\ell^{\prime \prime}-\ell+M_{L_{1}}{ }^{\prime}+M_{L_{1}}+S_{2_{1}}+S_{2}{ }^{\prime}-2 s+M S_{1}{ }^{\prime}+M_{S_{1}}} \\
& \phi_{2}^{\prime}, M_{L_{2}}, M_{\ell^{\prime \prime}, M_{L_{2}}} \\
& \mathrm{Ml}_{\ell} \mathrm{NS}_{2}, \mathrm{NS}_{2}{ }^{\prime}, \mathrm{N}_{\mathrm{S}} \\
& \left\{\left[L_{1}^{\prime}\right]\left[L_{1}\right]\left[S_{1}^{\prime}\right]\left[S_{1}\right]\right\}^{\frac{1}{2}} \times
\end{aligned}
$$




$$
\begin{aligned}
& \times\left(\begin{array}{ccc}
L_{2} & \ell^{\prime \prime} & L_{1}^{\prime} \\
M_{L_{2}} & M_{\ell^{\prime \prime}} & M_{L_{1}}^{\prime}
\end{array}\right)\left(\begin{array}{ccc}
S_{2}^{\prime} & s & S_{1}^{\prime} \\
N_{S_{2}} & N_{S} & N_{S_{1}^{\prime}}
\end{array}\right)\left(\begin{array}{ccc}
L_{2}^{\prime} & \ell & L_{1} \\
M_{L_{2}^{\prime}} & M_{\ell} & M_{L_{1}}
\end{array}\right)\left(\begin{array}{ccc}
S_{2}^{\prime} & s & S_{1} \\
N_{S_{2}}^{\prime} & N_{S} & N S_{1}
\end{array}\right) \\
& \times\left(\ell^{\mathrm{N}-3} \phi_{2}\left\{\mid \ell^{\prime \prime N-2_{\phi_{1}}}\right)<\mathrm{n} \ell^{\prime \prime} s, \mathrm{M}_{\ell}{ }^{\prime \prime} \mathrm{N}_{\mathrm{S}} \mid \ell s, \mathrm{M} \ell \mathrm{N}_{\mathrm{S}}>\right. \\
& <\ell_{n}^{N-3} \phi_{2} \mid \ell^{N-3} \phi_{2}> \\
& <\ell \mathrm{n}^{\mathrm{N}-3} \phi_{2}, \mathrm{n} \ell^{\prime \prime} \mathrm{s}, \phi_{1}^{\prime} \mid \ell^{N-3_{\phi_{1}}}>=\left(\ell^{N-3} \phi_{2}\left\{\mid \ell^{N-2_{\phi_{1}}}\right) \int_{0}^{\infty} f_{\ell}(r) F_{n} \ell^{\prime \prime}(r) \mathrm{dr}\right. \\
& \times\left[\int_{0}^{\infty} f_{\ell}(r) F_{\ell n}(r) d r\right]^{N-3} \delta\left(\phi_{1}, \phi_{1}^{\prime}\right) \delta\left(\phi_{2}, \phi_{2}^{\prime}\right) \dot{v}\left(\ell, \ell^{\prime \prime}\right) \delta(\ell n, \ell) .
\end{aligned}
$$

or

Of course, the results of Eq. (2d) could have been intuitively clear except for the coefficient in front of the integrals. In Eq. (2e) $f_{\ell}(r)$ is the radial wave function of one of the $\left|\ell N_{\phi_{0}}\right\rangle$ neutral ground state atoms; $f_{n \ell}(r)$ and $f_{\ell}$ are the wave functions of the excited runing electron and the corresponding core electrons of the $\mid \ell_{n}^{I J-3} \phi_{2}, n \ell$ "'s $\phi_{1}{ }^{\prime}>$ doubly ionized configuration. In Eqs. (2a) through (2c) the quantities $\left(\ell N^{\prime} \psi\left\{\mid \ell N^{\prime}-1 \phi\right)\right.$ are the coefficients of fractional parentage $(6)$ and the symbols in large parentheses are the usual 3 - j symbols.?

The probability that the doubly ionized atom will be in an excited state of the form $\left|\ell_{n}{ }^{\prime N-3} \phi_{2}, n \ell^{\prime \prime} s, \phi_{1}\right\rangle$ would be proportional to $\left|a_{n}\right|^{2}$, where

$$
a_{n}=\sum_{f} a_{n f}
$$

This means we have to calculate the quantities in braces of Eq. $(2 \mathrm{c})$ or, in other words, sum over expressions of the form $\left\langle f_{2} \mid \ell s M_{\ell} M_{S}\right\rangle$, which are the overlap integrals between the bound electronic states and all the possible final states of the free electron. We can, however, avoid these calculations by using the experimentally available total cross section data for removing two electrons from the atom. If we represent the double ionization cross section by $\mathrm{Q}^{++}(E)$, where $E$ represents the energy of the intial free electron state $\left|f_{j}\right\rangle$, then from Eqs. (2c), (2e), and (3a) 
we have for the excitation cross section of the state $\mid \ell N-3_{\phi_{2}}, \mathrm{n} \ell$ "s, $\left.\phi_{1}\right\rangle$ from the ground state $\mid \ell N_{\phi_{O}}>$, after impinging with an electron having a kinetic energy $E$,

$$
\begin{aligned}
& Q_{n}(E)=\left\{\sum _ { \phi } \left(\ell N-3_{\phi_{2}}\left\{\mid \ell N-2_{\left.\phi_{1}\right)(\ell} N-2_{\phi_{1}}\{\mid \ell N-1 \phi)\left(\ell N-1_{\phi}\left\{\mid N_{\phi_{0}}\right)\right.\right.\right.\right.
\end{aligned}
$$

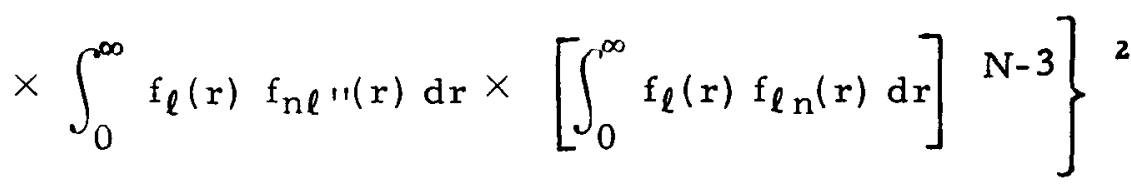

$$
\begin{aligned}
& Q^{++}(E) \delta\left(\ell, n \ell^{\prime \prime}\right) \delta(\ell, \ell n)
\end{aligned}
$$

where $Q_{n}(E)$ gives the excitation cross section for the $\mid \ell_{n}^{N-3} \phi_{2}, n \ell$ "s $\left.\phi_{1}\right\rangle$ configuration, the N-3 $\ell_{n}$ electrnns couple to give $\phi_{2}$ i.e., $L_{2}, S_{2}$; then these angular momenta couple with $\ell^{\prime \prime}$ to give $L_{1}, S_{1}, M_{1}, N_{1}$ or $\phi_{1}$. For the case of Noble gas atoms, which are of interest to this paper, $\ell=\ell_{n}=n \ell^{\prime \prime}=1, N=6$ and the only possible $L_{2} S_{2}$ states are ${ }^{2} \mathrm{D},{ }^{2} \mathrm{P}$ or ${ }^{4} S^{8}$ The possible final $L_{1} S_{1}$ states would be ${ }^{1} \mathrm{D},{ }^{3} \underline{P}$ or ${ }^{1} S$. This is seen from $\mathrm{Eq} \cdot(\mathrm{Ze})$. Moreover, the summation over $\phi$ in Eq. (3b) breaks down since in the case of $\ell^{5}$ only one possible $\phi$ state can exist, namely $\underline{P}$.

So far neither the coefficients of fractional parentage nor anything in Eqs. ( $3 b)$ or $(3 c)$ gives us any information about the total angular momentum $\mathrm{J}=\mathrm{L}+\mathrm{S}$ of the excited state or its excitation cross section. In the above example, for the noble gas atoms we find that the only possible excited states, when two electrons are removed from an outer $\mathrm{p}^{6}$ shell, are $\left|\ell^{\prime} \phi_{2}, \mathrm{n} \ell^{\prime \prime} \mathrm{s},{ }^{1} \mathrm{D}\right\rangle,\left|\ell^{3} \phi_{2}, \mathrm{n} \ell^{\prime \prime} \mathrm{s},{ }^{3} \mathrm{P}\right\rangle$, or $\left|\ell^{1}{ }^{3} \phi_{2}, \mathrm{n} \ell^{\prime \prime} \mathrm{s},{ }^{1} \mathrm{~S}\right\rangle$, with $\phi_{2}$ assuming any of the three possihle states of $\ell^{13}$. In this example, ${ }^{1} \mathrm{D}_{\mathrm{J}}$ has a $\mathrm{J}=2$ and ${ }^{1} \mathrm{~S}_{\mathrm{J}}$ has a $\mathrm{J}=0$, but in ${ }^{3} \mathrm{P}_{\mathrm{J}}, \mathrm{J}$ can assume the value of 1,2 or 0 . In general, to find the probability of excitation for any $J$ we have to change the order of coupling of the angular momenta of the expression in Eq. (2a) from LS to jj coupling, i.e.,

$$
\begin{aligned}
\left|\ell_{\phi_{O}}\right\rangle & =\sum_{\phi_{1}, \phi}\left(\ell ^ { N - 1 } \phi \{ | \ell ^ { N _ { \phi _ { O } } } ) \left(\ell N-2_{\phi_{1}}\left\{\mid \ell^{N-1} \phi\right)\right.\right. \\
& \times \mid \ell^{N-2} L_{1} S_{1} ; \ell_{1} s_{1}, L S ; \ell_{2} s_{2} L_{O} S_{O} J_{O}>,
\end{aligned}
$$


(4b)

$$
\begin{aligned}
& \left|\ell_{\phi_{0}}^{N}\right\rangle=\sum_{\phi_{0} \phi_{1}}\left(\ell ^ { N - 1 } \phi \{ | \ell ^ { N } \phi _ { 0 } ) \left(\ell ^ { N - 2 } \phi _ { 1 } \left\{\mid \ell^{N_{\phi}} \sum_{J_{0} J_{1} j_{1} j_{2}}\right.\right.\right. \\
& \left\{\left[L_{0}\right]\left[S_{0}\right][L][S][J]\left[J_{1}\right]\left[j_{1}\right]\left[j_{2}\right]\right\}^{\frac{1}{2}}\left\{\begin{array}{lll}
L & \ell_{2} & L_{0} \\
S & s_{2} & S_{0} \\
J & j_{2} & J_{0}
\end{array}\right\} \\
& \left\{\begin{array}{lll}
L_{1} & l_{1} & L \\
S_{1} & s_{1} & S \\
J_{1} & j_{1} & J
\end{array}\right] \times\left|l^{N-2} L_{1} S_{1} J_{1} ; \ell_{1} s_{1} j_{1}, L S J ; l_{2} s_{2} j_{2}, J_{0} M_{0}\right\rangle
\end{aligned}
$$

In the above equations $j_{n}=l_{n}+s_{n}$, the quantities in curly brackets are the 9 -j symbol, and to distinguish the two separated electrons, for the purpose of summation, a subscript is added. As in Eq. (2b) we can uncouple the eigenfunction $\mid \ell^{N-2} L_{1} S_{1} J_{1} ; l_{1} s_{1} j_{2}$ LSJ ; $l_{2} s_{2} j_{2}, J_{0} M_{0}>$ in terms of 3-j symbols and a product of the three wave functions, namely. $\left|\ell^{N-L_{1}} S_{1} J_{1} M_{1}>\right| \ell_{1} s_{1} j_{1} M_{j_{1}}>\mid \ell_{2} s_{2} j_{2} M_{j_{2}}>$. Using instead the $j j$ coupled eigenfunctions, Eq. (2c) becomes

$$
\sum_{f} a_{n f J_{1}}=\sum_{\phi_{q} \phi_{1}, J_{2} j_{1}, j_{2}}\left(l ^ { N - 2 } \phi _ { 1 } \{ | l ^ { N - 1 } \phi ) \left(l^{N-1} \phi\left\{\mid l^{N} \phi_{2}\right\}\right.\right.
$$

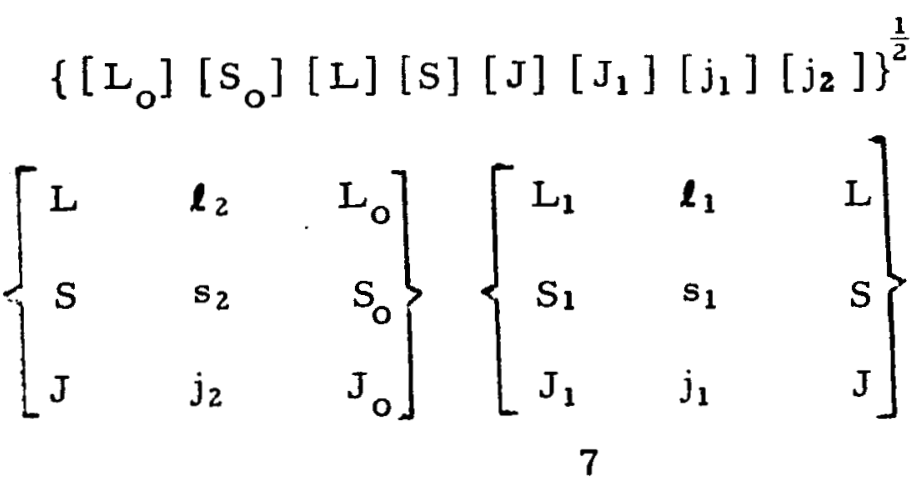




$$
x<\ell{ }_{n}^{\prime N-3} L_{2} S_{2} j_{2} ; n \ell^{\prime \prime} s, L_{1}^{\prime} S_{1}^{\prime} J_{1}^{\prime} M_{1}^{\prime} \mid \ell^{N-2} L_{1} S_{1} J_{1} M_{1}>\quad \times
$$

[ summation over all final states of the overlap integrals and some $3-j$ symbols].

In Eq. (4c) we have added an extra index $J_{1}$ to the coefficients anf to designate the total angular momentum of the excited state. As before, the last term in brackets is simply the overlap integrals and is proportional to the double ionization cross section, and the first portion gives the excitation probability. The excitation cross section to any state of the form $\mid \ell n^{N-3} L_{2} S_{2} J_{2} ; n \ell^{\prime \prime} s j, J_{1} M_{1}>$ from the ground state $\left.\right|_{\ell} \mathrm{N}_{\phi_{0}}>$ is,

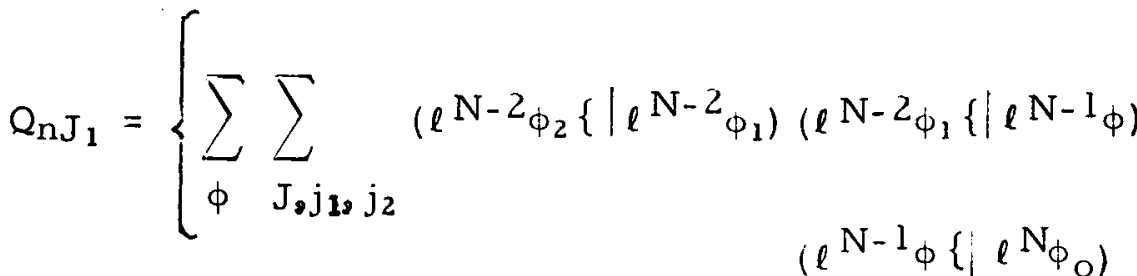

$$
\begin{aligned}
& \times\left\{\left[\mathrm{L}_{\mathrm{O}}\right]\left[\mathrm{S}_{\mathrm{O}}\right][\mathrm{L}][\mathrm{S}][\mathrm{J}]\left[\mathrm{J}_{1}\right]\left[\mathrm{j}_{1}\right]\left[\mathrm{j}_{2}\right]\right\}^{\frac{1}{2}} \\
& \left\{\begin{array}{lll}
L & \ell_{2} & L_{O} \\
S & s_{2} & S_{O} \\
J & j_{2} & J_{O}
\end{array}\right\}\left\{\begin{array}{lll}
L_{1} & \ell_{1} & L \\
S_{1} & s_{1} & S \\
J_{1} & j_{1} & J
\end{array}\right\} \quad \int_{0}^{\infty} f_{l}(1) f_{n \ell} 11(r) d r \\
& \left.\times\left[\int_{0}^{\infty} f_{\ell}(r) f_{\ell n}(r) d r\right]^{N-3}\right]^{2} Q^{++}(E)(\ell, \ell n) 0\left(\ell, n \ell l^{\prime \prime}\right) .
\end{aligned}
$$

For the calculations of the radial integrals, a self-consistant HartreeFock computer program in the Slater approximation was used, ${ }^{10}$ and the results for neon, argon, and krypton are given in Table I. As an illustration, we have calculated the excitation cross section for all the $3 \mathrm{p}^{3}-4 \mathrm{p}$ configuration of argon III, and this is presented in Table II. The total cross section for having a $3 \mathrm{p}^{3}-4 \mathrm{p}$ configuration afte $\mathrm{r}$ collision with a $100 \mathrm{ev}$ electron is about $0.88 \times 10^{-18}$, and this is divided among all the possible angular momentum states permissible from the $3 \mathrm{p}^{3}-4 \mathrm{p}$ configuration. 


\section{Case Two}

Here we assume that the atom loses one electron through collision, and assumes an excited configuration of the form $\left(\ell \mathrm{N}-2, \mathrm{n}_{1}\right)$. Then, through another collision, loses the excited $n l_{1}$ electron or one of the $\ell \mathrm{N}-2$ core electrons to as sume a final ( $\left.\ell^{\mathrm{N}-3}, \mathrm{n} \ell^{\prime \prime}\right)$ configuration, where $\mathrm{N}$ is the total number of electrons in the valence shell. Having seen the principle of the calculations for case one, we can now write the cross section without going through the detailed mathematics, the only difference being that one has to go twice through equations of the form Eq. (2c). Figure 2 shows schematically what happens.

In the second collision where the excited running electron is lost, we have for the excitation cross section of the state $\left|\ell_{2 m} \phi_{2}, \phi_{1}\right\rangle$

$$
\begin{aligned}
& Q_{1}\left(E, E^{\prime}\right)=\left\{\sum _ { \phi , \phi _ { 1 } } \left(\ell ^ { N - 3 } { } _ { 1 2 } \left\{\mid \ell^{\left.N-2_{\phi_{1}}\right)\left(\ell N-2_{\phi_{1}}\left\{\mid \ell^{N-1} \phi\right)\right.}\right.\right.\right. \\
& \left(\ell N-1+\left\{\ell^{N} \phi_{0}\right) \int_{0}^{\infty} f_{\ell}(r) f_{n \ell}(r) d r\right. \\
& \times\left[\int f_{\ell}\left(r^{\prime}\right) f_{\ell_{1 n}}\left(r^{\prime}\right) d r^{\prime}\right]^{N-2} \\
& \left.\times \int_{0}^{\infty} f_{\ell_{1 n}}\left(r^{\prime \prime}\right) f_{m \ell_{2}}\left(r^{\prime \prime}\right) d r \times\left[\int_{0}^{\infty} f_{\ell_{1 n}}(r) f_{\ell_{2 m}}(r) d r\right]^{N-3}\right]^{2} \\
& Q^{+}(E) Q_{n}^{+} \ell_{1}\left(E^{\prime}\right) \delta\left(\ell, \ell_{1 n}\right) \circlearrowleft(\ell, n \ell) \delta\left(\ell, m \ell_{2}\right) \delta\left(\ell, \ell_{2 m}\right)
\end{aligned}
$$

where $Q^{+}(E)$ is the usual single ionization cross section and $Q \mathrm{~N}_{1}\left(E^{\prime}\right)$ is the ionization cross section for removing the $n \ell_{1}$ excited electron from the $\left(\ell_{1 n}-Z_{n} \ell_{1}\right)$ configuration. $Q_{n} \ell_{1}$ could be very large as the excited $n \ell_{1}$ electron assumes higher orbits. The first set of the two integrals in Eq. (6) gives the overlap integrals for the bound states when after a collision $\ell N \rightarrow\left(\ell \mathrm{N}^{-2}, \mathrm{n} \ell_{1}\right)$, while the second set of the integrals give the overlap integrals for the $\left(\ell_{1 n}^{\mathrm{N}}-2, n \ell_{1}\right) \rightarrow\left(\ell_{1}^{\mathrm{N}} \mathrm{N}^{2}\right) \rightarrow\left(\ell_{2 \mathrm{~m}}^{\mathrm{N}-3}, \mathrm{~m} \ell_{2}\right)$ situation. The possible final states are exactly the same as those given in case one, namely, $\left|\ell^{3} \phi_{2} ; m \ell_{2} s, \phi_{1}\right\rangle$, where $\phi_{1}$ is a ${ }^{3} \mathrm{P},{ }^{1} \mathrm{D}$, or a ${ }^{1} S$ state. Tables III and IV give the radial integrals for the above two processes. 
Now, if in the second collision, instead of the excited electron a core electron is removed, $i . e$. , if $\left(\ell \mathrm{N}^{-2}, n \ell_{1}\right)$ goes into the intermediate state $\left(\ell \mathrm{N}^{-3}, \mathrm{n} \ell\right)$, the total orbital and spin angular momenta of the final states will not be evident as in the previous cases. We have to change the order of coupling of the electrons of the excited singly ionized atom. After having separated the electron which has to be lost from the core i.e., by expressing the state in terms of coefficients of fractional parentage,

$$
\begin{aligned}
\left|\ell_{1 \mathrm{n}}^{\mathrm{N}-2} \mathrm{~L}_{1} \mathrm{~S}_{1} ; \mathrm{n} \ell_{1} \mathrm{~s}, \mathrm{LSJ}\right\rangle & =\sum_{\phi_{2}}\left(\mathrm{P}^{\mathrm{N}-3} \phi_{2}\left\{\mid \mathrm{PN}-2_{\phi_{1}}\right)\right. \\
& \times \mid \ell_{1 \mathrm{n}}^{\mathrm{N}-3} \mathrm{~L}_{2} \mathrm{~S}_{2} ; \ell_{1 \mathrm{n}} \mathrm{s}, \mathrm{L}_{1} \mathrm{~S}_{1} ; \mathrm{n} \ell_{1} \mathrm{~s}, \mathrm{LSJ}>,
\end{aligned}
$$

we then change the order by first coupling the excited $n \ell_{1}$ s electron to the $L_{2} S_{2}$ core states and then couple the separated $\ell_{1 n}$ s core electron to the resultant states to obtain the final LS.S states, i.e.,

$$
\begin{aligned}
& \left|\ell_{1 \mathrm{n}}^{\mathrm{N}-\bar{z}} \mathrm{~L}_{1} \mathrm{~S}_{1} ; \mathrm{n} \ell_{1} \mathrm{~s}, \quad \mathrm{LSJ}\right\rangle=\sum_{\phi_{2}}\left(\mathrm { P } ^ { \mathrm { N } - 3 } \phi _ { 2 } \left\{\mid \mathrm{P}^{\left.\mathrm{N}-\mathcal{L}_{\phi_{1}}\right)} \sum_{\overline{\mathrm{L}} \overline{\mathrm{S}}}\left\{[\overline{\mathrm{L}}][\overline{\mathrm{S}}]^{\frac{1}{2}}\right.\right.\right.
\end{aligned}
$$

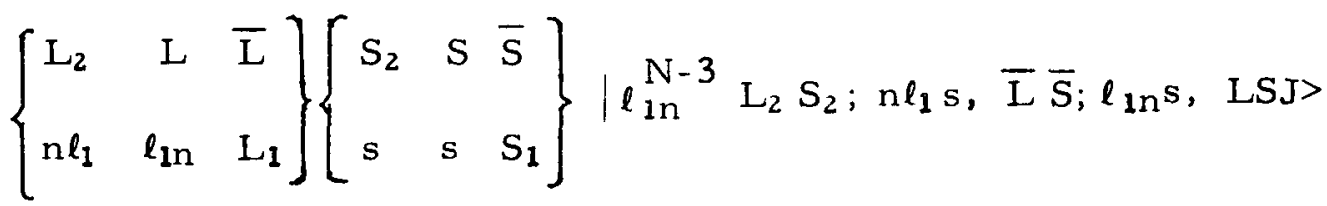

where the quantities in large curly brackets are the $6-j$ symbols. ${ }^{8}$ Now we use Eq. (7b) to calculate the overlap integrals between this singly ionized state and the doubly ionized states. The matrix element involved is,

$$
\begin{aligned}
& \therefore \ell_{1 \mathrm{n}}^{\mathrm{N}-2} \phi_{1} ; \mathrm{n} \ell_{1} \mathrm{~s}, \phi: F_{1 \mathrm{i}} \mid \ell_{2 \mathrm{~m}}^{\mathrm{N}-3} \phi_{2} ; \mathrm{m} \ell_{2} \phi_{1}^{\prime} ; F_{1 \mathrm{f}}, F_{2 \mathrm{f}}> \\
& =\left\{\alpha \sum_{f}\left\langle\ell_{1 n} s\right| F_{2 s}>\left\langle F_{1 i} \mid F_{1 f}\right\rangle\right\} \\
& \times \int_{0}^{\infty} f_{n \ell_{1}}(r) f_{m \ell_{3}}(r) d r \times\left[\int_{0}^{(\infty)} f_{\ell_{I n}}(r) f_{l_{m}}(r)\right]^{N-3} \\
& \delta\left(\phi_{1}, \bar{\phi}\right) \delta\left(\mathrm{n} \ell_{1}, m \ell_{2}\right) \delta\left(\ell_{1 n}, \ell_{2 m}\right)
\end{aligned}
$$


where $\alpha$ replaces all the $3-j$ symbols and summartions over them. It is seen that the only possible excited states are the $\bar{\phi}$ states, namely LS. From Eqs. (7b) and (7c) we can now write the excitation cross section for all the states $\left|\ell_{3 \mathrm{~m}}^{\mathrm{N}} \phi_{2}, \mathrm{ml}_{3} \mathrm{~s} \phi\right\rangle$, where $\bar{\phi}$ stands for $\bar{L} \bar{S}$,

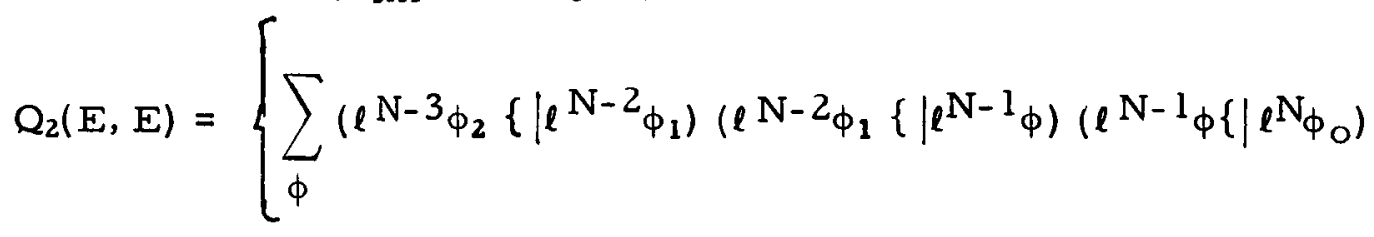

$$
\begin{aligned}
& {[\overline{\mathrm{L}}]^{\frac{1}{2}}[\overline{\mathrm{S}}]^{\frac{1}{2}}\left\{\begin{array}{ccc}
\mathrm{L}_{2} & \mathrm{~L} & \overline{\mathrm{L}} \\
\mathrm{n} \ell_{1} & \ell_{1 \mathrm{n}} & \mathrm{L}_{1}
\end{array}\right\}\left\{\begin{array}{ccc}
\mathrm{S}_{2} & \mathrm{~S} & \overline{\mathrm{S}} \\
\mathrm{s} & \mathrm{s} & \mathrm{S}_{1}
\end{array}\right\}} \\
& \times \int_{0}^{\infty} f_{\ell}(r) f_{n \ell_{l}}(r) d r \times\left[\int_{0}^{\infty} f_{\ell}\left(r^{\prime}\right) f_{\ell}\left(r^{\prime}\right) d r\right]^{N-2} \\
& \left.\times \int_{0}^{\infty} f_{n l_{1}}\left(r^{\prime \prime}\right) f_{m l_{3}}\left(r^{\prime \prime}\right) d r^{\prime \prime} \times\left[\int_{0}^{\infty} f_{l_{1 n}}(r) f_{\ell_{3 m}}(r) d r\right]^{N-3}\right\}^{2} \\
& Q^{+}(E) Q_{l_{1 n}}^{+}\left(E^{\prime}\right)
\end{aligned}
$$

There $Q_{\ell_{1 n}}^{+}(E)$ is the cross section for removing an electron from the core of the singly ionized excited state of the ( $\left.\ell_{\text {in }}^{N}, 2, n \ell_{1}\right)$ configuration. This cross section is proportional to the square-absolute.value of the overlap integrals in Eq. (7c) between bound and the free electrons. Table $\mathrm{V}$ gives the overlap integrals for the bound atomic states of Eq. (7c) for neon, argon, and krypton and Tables VI and VII gives the necessary coefficients of fractional parentage for the $\mathrm{p}^{\mathrm{N}}$ shells of the Noble gas atoms.

For the case of Noble gas atoms, we can also determine the possible $\bar{\phi}$ states in Eq. (7a) $\mathrm{L}=1, \mathrm{~S}=1 / 2, \phi_{2}$ is either ${ }^{4} \mathrm{~S},{ }^{2} \mathrm{P}$ or ${ }^{2} \mathrm{D}$. From the triangular relations of the $6-j$ symbols $\bar{\phi}$ can be ${ }^{3} \mathrm{P}$ when $\phi_{2}={ }^{4} \mathrm{~S},{ }^{1,3}[\mathrm{~S}, \mathrm{P}, \mathrm{D}]$ when $\phi_{2}={ }^{2} \mathrm{P}$ and finally $\bar{\phi}={ }^{1,3}[\mathrm{P}, \mathrm{D}]$, when $\phi_{2}={ }^{2} \mathrm{D}$. 


\section{CONCLUSIONS}

Two cases of excitation have been discussed. For case one, since the double ionization cross section is large ${ }^{11}$, i.e., of the order of $10^{-17} \mathrm{~cm}^{2}$ and because of the relatively large overlap integrals, we notice that removing two electrons from the valence shell causes an electron to go to an excited nl" shell with a relatively high probability. From Table I we see that this probability decreases as $n$ increases.

The probability of the excited electron to go to continum, that is to say the atom to be triply ionized, after the removal of two electrons, would be ( 1 - $\sum$ probability to go to $n l "$ excited shell). For the case of neon, we have $Q^{+++}(E)=0.17 Q^{+++}(E)$, for argon $Q^{+++}(E)=0.045 Q^{+++}$ (E) and for krypton $\mathrm{Q}^{+++}(E)=0.04 \mathrm{Q}^{++}(E) \cdot \mathrm{Q}^{+++}$is the cross section for triply ionizing the atom. It is seen that in the measurements of ionization cross section of removing two electrons could possibly cause the atom to be rather triply ionized.

For case two, we treated two special situations. Both of these situations are not likely to occur in discharges of Noble gas atoms since the lifetime of the lower excited states are relatively short; however, in atorns where the excited states have longer lifetimes this situation could become probable. An example is the $3 \mathrm{p}^{4}[3 \mathrm{p}]-3 \mathrm{~d}^{2} \mathrm{~F}$ states of argon. In this case, the atom becomes ionized and excited and then relaxes to the above ionized metastable state. If the population of these states is large, then it is quite possible that the atom becomes doubly ionized through these states. Labuda, et. al. ${ }^{12}$, have measured the population density of the se states and found them to be quite large. 


\section{REFER ENCES}

1. Koozekanani, S.H., "Excitation Cross Section of Some of the States of NeII, ArII and KrII by Electron Collision," Jo of Quantum Electronics, Dec., 1966.

2. Bennett, W.R., Jr., et.al,, "Direct Electron Excitation Cross Sections Pertinent to the Argon Ion Laser, " Physical Review Letters, Vol. 17, No. 19, p. 987, Nov. 1966.

3. Bennett, W.R., Jr., "Inversion Mechanisms in Gas Lasers," Applied Optics Supplement 2 of Chemical Lasers, p. 3-33, 1965.

4. Lamb, W. E., Jr. and Skinner, M. , "The Fine Structure of Singly Ionized Helium," Phys. Rev., Vol. 78, No. 5, pp.539..550, 1 June 1959.

5. Schiff, L. I., Quantum Mechanics, Second Edition (1955), McGrawHill Book Co.

6. Nielson, C.W. and Koster, G. F., "Spectroscopic Coefficients for $\mathrm{p}^{\mathrm{n}}, \mathrm{d}^{\mathrm{n}}$ anc $\mathrm{f}^{\mathrm{n}}$ Configurations," MIT Press, Cambridge, Mass., 1963. Also B. R. Judd, Operator Techniques in Atomic Spectroscopy, McGraw-Hill Book Co., Inc, 1963.

7. Rotenberg, M.R., Bivins, N. Metropslis and J.R. Wooten, The 3-J and 6-j Symbols, MIT Tech. Press, Cambridge, Mass., 1959.

8. Condon, E. V. and Shortley, G. H., The Theory of Atomic Spectra, Cambridge University Press, 1935.

9. Edmonds, A. R, Angular Momentum in Quantum Mechanics, University Press, Princeton, 1957.

10. Herman, F. and Skillman, S. Atomic Structure Calculations, PrenticemHall, Inc, Englewood Cliffs, New Jersey, 1963.

11. Kieffer, L. J., Dunn, G. H., "Electron Impact Ionization Cross Section Data for Atoms, Atomic Ions and Diatomic Molecules $I$. Experimental Data," Rev. of Modern Phys., Vol. 38, No. 1, 1966.

12. Labuda, E. F. , Gordon, E. I., and Miller, R. C., J. of Quantum Electronics, QEm-1, 273 (1965). 
ACKNOWLEDGEMENT

The author wishes to thank Dr. C. Levis for reading the manuscript and Mr. H.C. Friedman for computational assistance. 

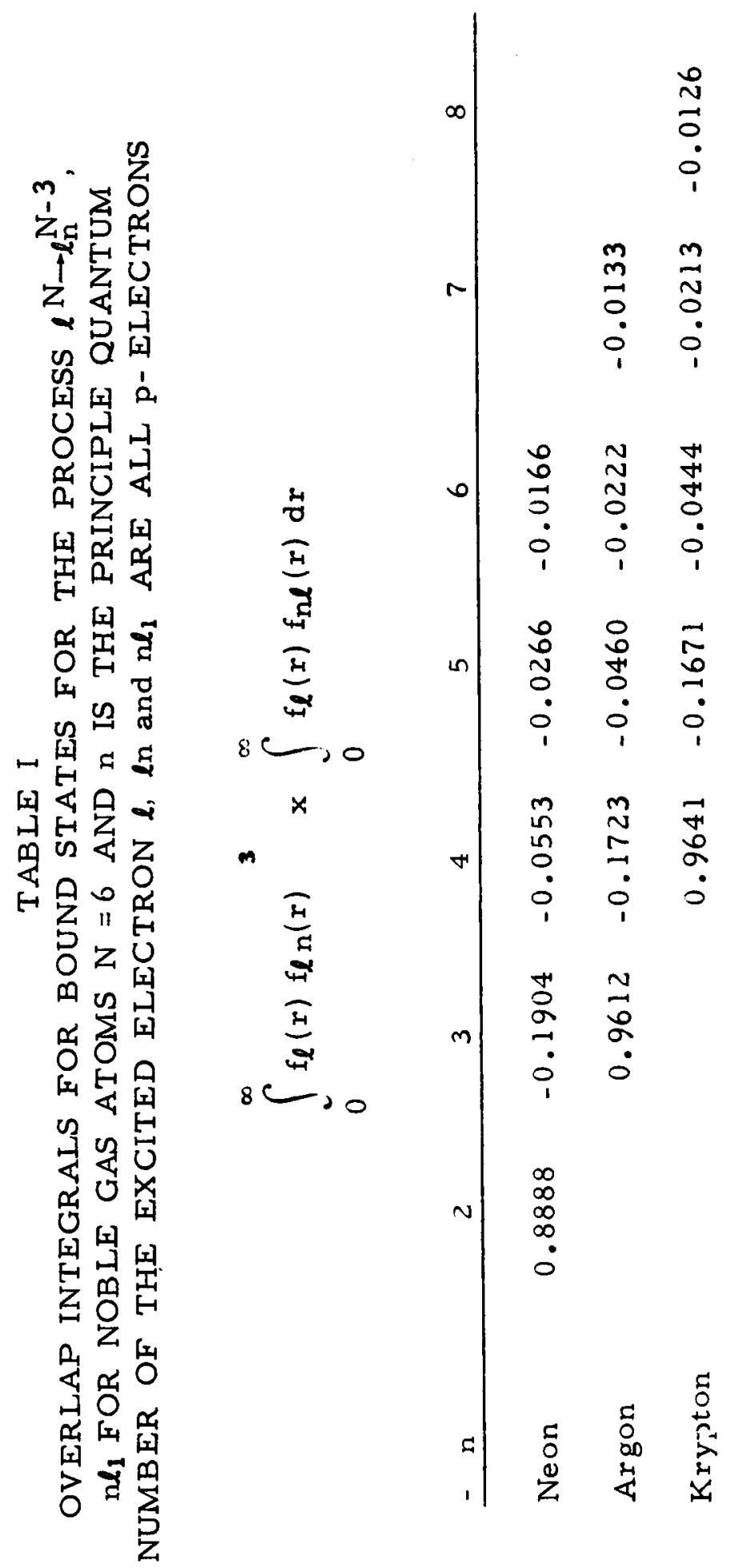
TABLE II

EXCITATION CROSS SECTION FOR $\left|\mathrm{p}^{3} \phi_{2}, 4 \mathrm{p} \phi_{1}\right\rangle$ STATES OF ARGON ${ }^{++}$FOR CASE ONE AT ELECTRON ENERGIES OF ABOUT $100 \mathrm{EV}$. $\mathrm{Q}^{++}(100 \mathrm{ev})=2.96 \times 10^{-17} \mathrm{~cm}^{2}(\operatorname{ref~} 10)$

$$
\begin{aligned}
& \mathrm{Q}\left(\mid\left(3 \mathrm{p}^{3}\right)^{4} \mathrm{~S}, 4 \mathrm{p},{ }^{1} \mathrm{D}_{2}>\right)=2.17 \times 10^{-19} \mathrm{~cm}^{2} \\
& Q\left(1\left(3 \mathrm{p}^{3}\right)^{4} \mathrm{~s}, 4 \mathrm{p},{ }^{3} \mathrm{P}_{2}>\right)=0.95 \times 10^{-19} \mathrm{~cm}^{2} \\
& \mathrm{Q}\left(\mid\left(3 \mathrm{p}^{3}\right) \mathrm{S}, 4 \mathrm{p},{ }^{3} \mathrm{P}_{1}>\right)=0.57 \times 10^{-19} \mathrm{~cm}^{2} \\
& \mathrm{Q}\left(\left(3 \mathrm{p}^{3}\right)^{4} \mathrm{~S}, 4 \mathrm{p},{ }^{3} \mathrm{P}_{\mathrm{O}}>\right)=0.19 \times 10^{-19} \mathrm{~cm}^{2} \\
& \left.Q\left(\mid 3 \mathrm{p}^{3}\right)^{2} \mathrm{D}, 4 \mathrm{p},{ }^{3} \mathrm{P}_{2}>\right)=1.20 \times 10^{-19} \mathrm{~cm}^{2} \\
& \mathrm{Q}\left(\mid\left(3 \mathrm{p}^{3}\right)^{2} \mathrm{D}, 4 \mathrm{p},{ }^{3} \mathrm{P}_{1}>\right)=0.72 \times 10^{-19} \mathrm{~cm}^{2} \\
& Q\left(\mid\left(3 \mathrm{p}^{3}\right)^{2} \mathrm{D}, 4 \mathrm{p},{ }^{3} \mathrm{P}_{\mathrm{\sigma}}\right)=0.25 \times 10^{-19} \mathrm{~cm}^{2} \\
& Q\left(\mid\left(3 \mathrm{p}^{3}\right)^{2} \mathrm{p}, 4 \mathrm{p},{ }^{1} \mathrm{D}_{2}>\right)=0.72 \times 10^{-19} \mathrm{~cm}^{2} \\
& Q\left(\mid\left(3 p^{3}\right)^{2} \mathrm{P}, 4 \mathrm{p},{ }^{3} \mathrm{P}_{2}>\right)=0.72 \times 10^{-19} \mathrm{~cm}^{2} \\
& \left.Q\left(\mid\left(3 \mathrm{p}^{3}\right)^{2} \mathrm{P}, 4 \mathrm{p}\right),{ }^{3} \mathrm{P}_{1}>\right)=0.42 \times 10^{-19} \mathrm{~cm}^{2} \\
& Q\left(\mid\left(3 \mathrm{p}^{3}\right)^{2} \mathrm{P}, 4 \mathrm{p},{ }^{3} \mathrm{P}_{\mathrm{O}}>\right)=0.13 \times 10^{-19} \mathrm{~cm}^{2} \\
& Q\left(\mid\left(3 \mathrm{p}^{3}\right)^{2} \mathrm{P}, 4 \mathrm{p},{ }^{1} \mathrm{~S}_{\mathrm{O}}>\right)=0.57 \times 10^{-19} \mathrm{~cm}^{2}
\end{aligned}
$$


OVERLAP INTEGRALS FOR THE PROCESS $\boldsymbol{l}^{\mathrm{N}} \rightarrow \boldsymbol{l}_{\mathrm{n}}^{\mathrm{N}-\boldsymbol{2}}, \mathrm{n} \boldsymbol{\ell}_{1}$

$\left[\int_{0}^{\infty} f_{\ell}(r) f_{\ell n}(r) d r\right]^{4} \times \int_{0}^{\infty} f_{\ell}(r) f_{n \ell_{1}}(r) d r$

$a_{2}$

$a_{3}$

$a_{4}$

$a_{5}$

$a_{6}$

$a_{z}$

Neon

0.980

$-0.1308$

$-0.0525-0.0290$

Argon

0.985

$-0.1195$

$-0.0466$

$-0.0247$

Krypton

$\begin{array}{llll}0.985 & -0.1165 & -0.04515 & -0.0237\end{array}$ 
TABLE IV

OVERLAP INTEGRAI FOR THE CASE

$\left(\ell_{1 \mathrm{n}}^{N-2}, \mathrm{n} \ell_{1}\right) \rightarrow \ell_{1 \mathrm{n}}^{N-2} \rightarrow \ell_{2 \mathrm{~m}}^{N-3}, \mathrm{n} \ell_{2}$

$\left[\int_{0}^{\infty} f_{\ell_{2} m}(r) f_{\ell_{1} n}(r) d r\right]^{3} \times \int_{0}^{\infty} f_{\ell_{1 n}}(r) f_{m \ell_{2}}(r) d r$

$\mathrm{Ne}$ II $2 \mathrm{p}^{3}-2 \mathrm{p} \quad 2 \mathrm{p}^{3}-3 \mathrm{p} \quad 2 \mathrm{p}^{3}-4 \mathrm{p} \quad 2 \mathrm{p}^{3}-5 \mathrm{p} \quad 2 \mathrm{p}^{3}-6 \mathrm{p}$

$\begin{array}{llllll}2 \mathrm{p}^{4}-2 \mathrm{p} & 0.9894 & -0.1177 & -0.0452 & -0.0247 & -0.0153 \\ 2 \mathrm{p}^{4}-3 \mathrm{p} & 0.9996 & -0.0476 & -0.0227 & -0.0133 & -0.0088 \\ 2 \mathrm{p}^{4}-4 \mathrm{p} & 0.9992 & -0.0416 & -0.0213 & -0.0128 & -0.0086 \\ 2 \mathrm{p}^{4}-5 \mathrm{p} & 0.9992 & -0.0397 & -0.0209 & -0.0127 & -0.0085 \\ 2 \mathrm{p}^{4}-6 \mathrm{p} & 0.9992 & -0.0390 & -0.0207 & -0.0126 & -0.0085\end{array}$

ArII $\quad \begin{array}{r}3 p^{3}-3 p \quad 3 p^{3}-4 p \quad 3 p^{3}-5 p \quad 3 p^{3}-6 p \quad 3 p^{3}-7 p \\ \hline\end{array}$

$\begin{array}{llllll}3 p^{4}-3 p & 0.9912 & -0.1072 & -0.0398 & -0.0208 & -0.0130\end{array}$

$\begin{array}{llllll}3 p^{4}-4 p & 0.9996 & -0.0435 & -0.0205 & -0.0116 & -0.0075\end{array}$

$\begin{array}{llllll}3 \mathrm{p}^{4}-5 \mathrm{p} & 0.9992 & -0.0350 & -0.0190 & -0.0113 & -0.0074\end{array}$

$\begin{array}{llllll}3 \mathrm{p}^{4}-6 \mathrm{p} & 0.9992 & -0.0323 & -0.0184 & -0.0111 & -0.0074\end{array}$

$\begin{array}{llllll}3 \mathrm{p}^{4}-7 \mathrm{p} & 0.9988 & -0.0311 & -0.0182 & -0.0111 & -0.0074\end{array}$

KrII $4 p^{3}-4 p \quad 4 p^{3}-5 p \quad 4 p^{3}-6 p \quad 4 p^{3}-7 p \quad 4 p^{3}-8 p$

$\begin{array}{llllll}4 \mathrm{p}^{4}-4 \mathrm{p} & 0.9980 & -0.1035 & -0.0384 & -0.0201 & -0.0123\end{array}$

$\begin{array}{llllll}4 \mathrm{p}^{4}-5 \mathrm{p} & 0.9996 & -0.0417 & -0.0198 & -0.0112 & -0.0071\end{array}$

$\begin{array}{llllll}4 p^{4}-6 p & 0.9992 & -0.0329 & -0.0182 & -0.0108 & -0.0071\end{array}$

$\begin{array}{llllll}4 \mathrm{p}^{4}-7 \mathrm{p} & 0.9988 & -0.0297 & -0.0176 & -0.0107 & -0.0071\end{array}$

$\begin{array}{llllll}4 p^{4}-8 p & 0.9988 & -0.0284 & -0.0173 & -0.0107 & -0.0071\end{array}$ 
TABLE V

OVERLAP INTEGRALS FOR THE CASE

$$
\begin{aligned}
& \left(\ell_{1 \mathrm{n}}^{\mathrm{N}-2}, \mathrm{n} \ell_{1}\right) \rightarrow\left(\ell_{1 \mathrm{n}}^{\mathrm{N}-3}, \mathrm{n} \ell_{1}\right) \rightarrow\left(\ell_{3 \mathrm{~m}}^{\mathrm{N}-3}, \mathrm{~m} \ell_{3}\right) \\
& \int_{0}^{\infty} f_{\ell_{1} \mathrm{n}}(r) f_{\ell_{3}} \mathrm{~m}(r) d r^{3} \times \int_{0}^{\infty} f_{n \ell_{1}}(r) f_{m \ell_{3}}(r) d \sigma
\end{aligned}
$$

\begin{tabular}{|c|c|c|c|c|c|}
\hline ArII & $2 p^{3}-3 p$ & $2 p^{3}-4 p$ & $2 p^{3}-5 p$ & $2 p^{3}-6 p$ & $2 p^{3}-7 p$ \\
\hline $3 p^{4}-3 p$ & 0.9912 & -0.1072 & -0.0398 & -0.0208 & -0.0130 \\
\hline $3 p^{4}-4 p$ & -- & .9477 & -.3402 & -0.0768 & -0.0438 \\
\hline $3 p^{4}-5 p$ & -- & - & .8444 & -0.5102 & -0.0653 \\
\hline $3 p^{4}-6 p$ & -- & - & -- & 0.6828 & -0.664 \\
\hline $3 p^{4}-7 p$ & -- & $\ldots$ & $\ldots$ & - & 0.4702 \\
\hline
\end{tabular}

$\begin{array}{rccccc}\text { NeII } & 2 \mathrm{p}^{3}-2 \mathrm{p} & 2 \mathrm{p}^{3}-3 \mathrm{p} & 2 \mathrm{p}^{3}-4 \mathrm{p} & 2 \mathrm{p}^{3}-5 \mathrm{p} & 2 \mathrm{p}^{3}-6 \mathrm{p} \\ 2 \mathrm{p}^{4}-2 \mathrm{p} & 0.9894 & -0.1177 & -0.0452 & -0.0247 & -0.0153 \\ 2 \mathrm{p}^{4}-3 \mathrm{p} & -- & 0.9341 & -0.3680 & -0.0842 & -0.0477 \\ -2 \mathrm{p}^{4}-4 \mathrm{p} & -- & -- & 0.8134 & -0.5405 & -0.0704 \\ 2 \mathrm{p}^{4}-5 \mathrm{p} & -- & -- & -- & 0.6378 & -0.7021 \\ 2 \mathrm{p}^{4}-6 \mathrm{p} & -- & -- & -- & -- & 0.4223\end{array}$

KrII ${ }^{\text {KrIII }} 4 p^{3}-4 p \quad 4 p^{3}-5 p \quad 4 p^{3}-6 p \quad 4 p^{3}-7 p \quad 4 p^{3}-8 p$

$\begin{array}{lccccc}4 p^{4}-4 p & 0.9920 & -0.1035 & -.0389 & -0.0201 & -0.0123 \\ 4 p^{4}-5 p & -- & 0.9555 & -0.3329 & -0.0742 & -0.0425 \\ 4 p^{4}-6 p & -- & -- & 0.8527 & -0.4794 & -0.0634 \\ 4 p^{4}-7 p & -- & -- & -- & 0.6954 & -0.6557 \\ 4 p^{4}-8 p & -- & - & -- & -- & 0.4919\end{array}$


COEFFICIENTS OF FRACTIONAL PARENTAGE FOR STATES $\mathrm{p}^{5} \phi$ WITH $\mathrm{p}^{4} \phi_{1}$ PARENTS

\begin{tabular}{|c|c|c|c|}
\hline $\mathrm{p}^{5} \phi / \mathrm{p}^{4} \phi_{1}$ & ${ }^{1} \mathrm{~S}$ & ${ }^{3} \mathrm{P}$ & ${ }^{1} \mathrm{D}$ \\
\hline${ }^{2} \mathrm{P}$ & $\sqrt{\frac{1}{15}}$ & $\sqrt{\frac{3}{5}}$ & $\sqrt{\frac{1}{3}}$ \\
\hline
\end{tabular}

COEFFICIENTS OF FRACTIONAL PARENTAGE FOR STATES $\mathrm{p}^{4} \phi_{1}$ WITH $\mathrm{p}^{3} \phi_{2}$ PARENTS

\begin{tabular}{lccc}
$\mathrm{p}^{4} \phi_{1} / \mathrm{p}^{3} \phi_{3}$ & ${ }^{4} \mathrm{~S}$ & ${ }^{2} \mathrm{P}$ & ${ }^{2} \mathrm{D}$ \\
\hline${ }^{3} \mathrm{P}$ & $\sqrt{\frac{1}{3}}$ & $-\frac{1}{2}$ & $-\frac{1}{2} \sqrt{\frac{5}{3}}$ \\
${ }^{1} \mathrm{D}$ & & $-\frac{1}{2}$ & $\sqrt{\frac{3}{2}}$ \\
${ }^{1} \mathrm{~S}$ & & 1
\end{tabular}



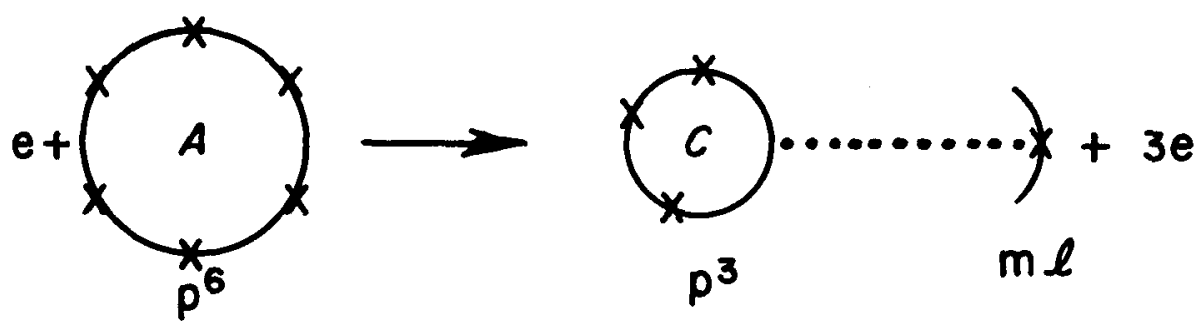

Fig. 1. An electron colliding with a neutral Noble gas atom resulting in three free electrons and an excited doubly ionized atom. For argon if the colliding electron has a kinetic energy of $100 \mathrm{ev}$ the cross section for the $3 p^{3}-4 p$ configuration is

$8.9 \times 10^{-19} \mathrm{~cm}^{2}$. 


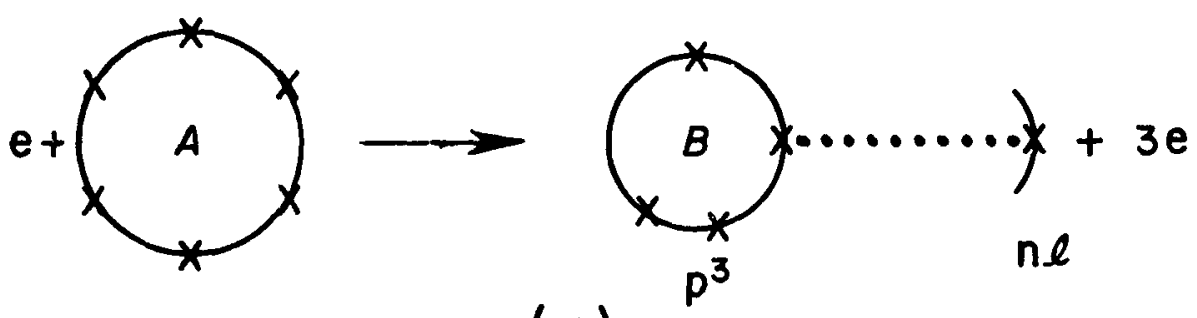

(a)
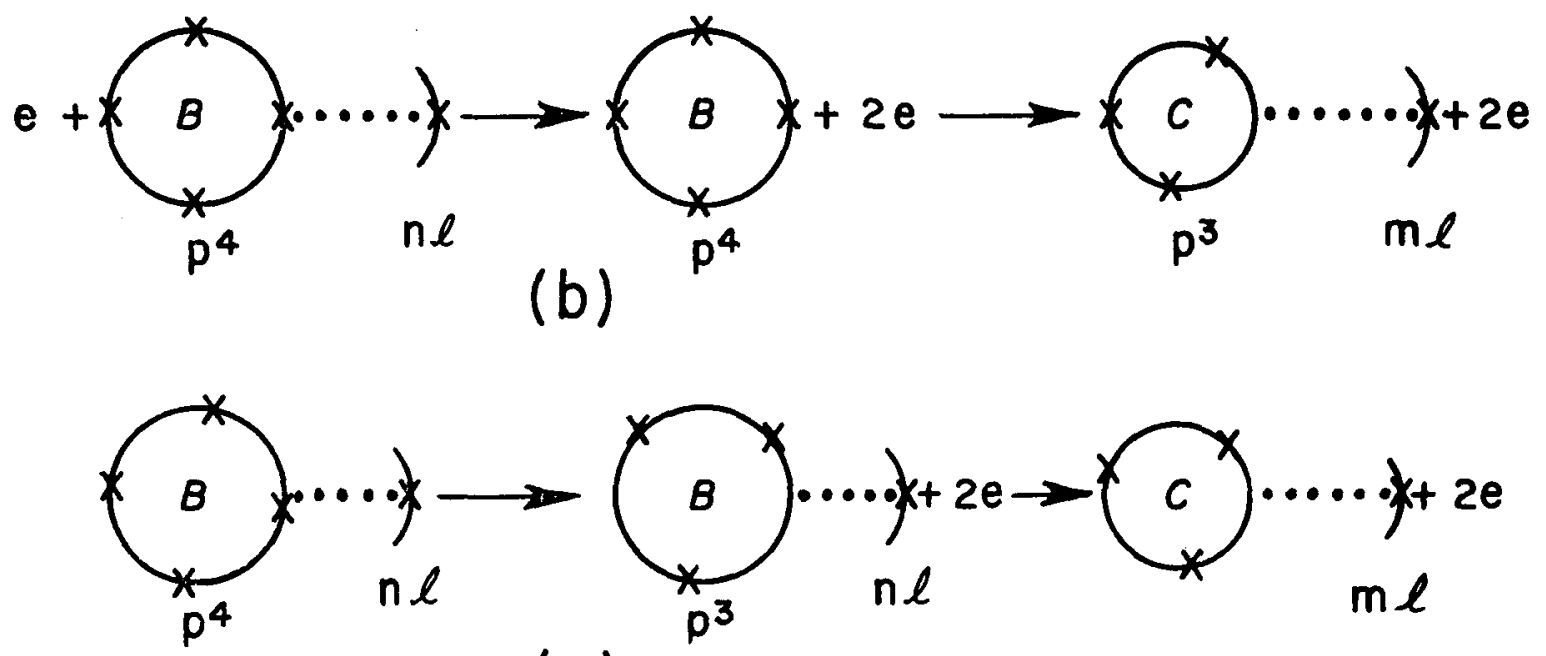

(c)

Fig. 2. Here the process of excitation is in two steps.

(a) The atom loses an electron and becomes excited to a $\mathrm{p}^{4}-\mathrm{n} \ell$ configuration.

(b) The excited ionized atom makes another collision, loses its excited electron and then relaxes to a doubly ionized excited state.

(c) The excited ionized atom through collision loses one of its core electrons and then relaxes to a doubly ionized excited state. 